\title{
Efecto in vitro de los suplementos vitamínicos que se venden como afrodisíacos sobre la movilidad espermática
}

\section{In vitro effect of Vitamin Supplements Sold as Aphrodisiacs on Sperm Motility}

\author{
Yulieth Catherine Quintero Quinchia ${ }^{1}$ Walter D. Cardona Maya ${ }^{1}$ \\ ${ }^{1}$ Grupo Reproducción, Departamento de Microbiología y \\ Parasitología, Facultad de Medicina, Universidad de Antioquia, \\ Medellín, Colombia
}

Urol Colomb 2019;28:177-182.

\section{Resumen \\ Palabras Clave \\ - afrodisíaco \\ - espermatozoides \\ - suplementos vitamínicos \\ - fertilidad \\ - vitaminas \\ - semen}

\section{Abstract}

Objetivo El objetivo de este trabajo es determinar el efecto in vitro de los suplementos vitamínicos que se venden como afrodisíacos sobre la movilidad espermática.

Materiales y métodos Comprimidos de venta libre obtenidos en diferentes centros de venta de productos naturales y sexuales de la ciudad de Medellín fueron evaluados (Sex Bull, Ses-Max, Vigorday, Up12, Furumbao, Canguro, MAGYMas) y diluidos en solución salina. Posteriormente, fueron mezclados en proporciones iguales con las muestras de semen de 22 voluntarios aparentemente sanos y se determinó el efecto sobre la movilidad en función del tiempo (0, 60, 120 y 180 minutos).

Resultados El análisis del efecto de los tratamientos con extractos acuosos de los suplementos vitamínicos sobre los espermatozoides, fueron divididos según la presencia de L-arginina en su composición. No se observaron cambios positivos sobre la movilidad espermática.

Conclusión Los suplementos vitamínicos que se venden en las tiendas naturistas y sexuales de la ciudad, no ejercen ningún efecto sobre la calidad seminal in vitro, seguramente basados en su composición solo sirven como suplementos energéticos.

Aim to determine the in vitro effect of the vitamin supplements sold as aphrodisiacs on sperm motility. received

September 4, 2017

accepted

October 3, 2017
DOI https://doi.org/

10.1055/s-0038-1651513.

ISSN 0120-789X.

eISSN 2027-0119.
Copyright $($ C 2019, Sociedad Colombiana License terms de Urología. Publicado por Thieme Revinter Publicações Ltda., Rio de Janeiro, Brazil. Todos los derechos reservados.

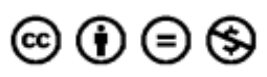


Keywords

- aphrodisiacs

- human spermatozoa

- vitamin supplements

- fertility

- vitamins

- semen
Materials and Methods The tablets of free sale obtained in different and sex shop from Medellín city were evaluated (Sex Bull, Ses-Max, Vigorday, Up12, Furumbao, Canguro, MAGYMas) and diluted in saline solution. Later, the semen samples from 22 apparently healthy volunteers were mixed in equal proportions and the effect on motility over time $(0,60,120$ and 180 minutes) was determined.

Results Analysis of the effect of treatments with aqueous extracts of vitamin supplements on spermatozoa were divided according to the presence of t-arginine in their composition. There were no positive changes in sperm motility.

Conclusion The vitamin supplements sold in the naturopathic shops and sexual stores of the city do not exert any effect on seminal quality in vitro, probably based on their composition only serve as an energetic supplement.

\section{Introducción}

Un afrodisiaco es una sustancia que es utilizada para el tratamiento de la disfunción eréctil o para mejorar el comportamiento sexual, ${ }^{1}$ según su mecanismo de acción se clasifican en los que aumentan la libido, la potencia o el placer sexual, ${ }^{2}$ varias formulaciones afrodisíacas están disponibles en el mercado con el fin de mejorar el deseo sexual en los hombres, aunque en la mayoría de los casos la seguridad y la eficacia de los medicamentos no ha sido demostrada. Ninguna sustancia vegetal, a excepción de la yohimbina, ha sido aprobada por la Administración de Alimentos y Medicamentos de los Estados Unidos (FDA), como medicación para la disfunción sexual masculina, ${ }^{3} \sin$ embargo, los derivados de plantas y mezclas naturales continúan siendo una alternativa popular para el tratamiento de los desórdenes sexuales. ${ }^{4}$

La mayoría de plantas reportadas como afrodisiacas son de origen y uso en países de Oriente y África y en menor proporción Europa y América, lo que puede estar determinado por la permanencia de medicinas tradicionales, especialmente en países asiáticos. ${ }^{5}$

En la literatura científica se ha evidenciado el uso de las plantas afrodisíacas para el mejoramiento de la concentración espermática, ${ }^{6}$ la espermatogénesis ${ }^{7}$ y la movilidad espermática tanto in vivo ${ }^{8}$ como in vitro, ${ }^{9,10}$ aunque también se ha reportado la inhibición de la movilidad espermática in vitro con el tratamiento de extractos de frutas afrodisíacas. ${ }^{11}$

La movilidad espermática es un componente central de la fertilidad masculina, los resultados de las técnicas de reproducción asistida han demostrado que las tasas de fertilización y de embarazos son pobres cuando los espermatozoides utilizados poseen movilidad baja comparado con los espermatozoides con movilidad normal. ${ }^{12}$ La disfunción espermática es una de las causas comúnmente observadas en los casos de infertilidad, su manifestación principal es la movilidad espermática por debajo del límite inferior de referencia propuesto en 2010 por la Organización Mundial de la Salud, ${ }^{13}$ lo que repercute negativamente en la probabilidad de concepción. ${ }^{14}$

Dentro de las causas de astenozoospermia se encuentra el óxido nítrico, un radical libre del nitrógeno que en concentraciones altas puede inducir la peroxidación de los lípidos de la membrana del espermatozoide ocasionando daño en el ADN y pérdida de la movilidad. ${ }^{15}$ El óxido nítrico desempeña un papel fundamental en la erección del pene, ${ }^{16}$ y algunas plantas y sustratos como L-arginina se encuentran en los medicamentos afrodisiacos con el fin de incrementar el óxido nítrico. El objetivo de este trabajo es determinar el efecto in vitro de los suplementos vitamínicos que se venden como afrodisíacos sobre la movilidad espermática.

\section{Materiales y Métodos}

\section{Muestras de semen}

Se emplearon 22 muestras de semen de voluntarios aparentemente sanos, mayores de 18 años con parámetros seminales normales según los lineamientos establecidos por la Organización Mundial de la Salud (OMS) en 2010. ${ }^{13}$ Las muestras de semen fueron recolectadas por masturbación en un recipiente estéril, después de una abstinencia sexual de 3 a 5 días y analizadas máximo 2 horas después de su recolección.

Suplementos vitamínicos/afrodisíacos usados en el estudio 1.

Se utilizaron los siguientes comprimidos de venta libre obtenidos en diferentes centros de venta de productos naturales y sexuales de la ciudad de Medellín: Sex Bull, Ses-Max, Vigorday, Up12, Furumbao, Canguro y MAGYMas.

Preparación de los extractos acuosos de los suplementos vitamínicos.

Cada suplemento vitamínico fue macerado completamente y disuelto en tampón fosfato salino (PBS, Gibco, Grand Island, NY, EEUU), a una concentración de $2.0 \mathrm{mg} / \mathrm{mL}$. Antes de su utilización como tratamiento sobre los espermatozoides se agitaron en vortex y fueron incubados a $35^{\circ} \mathrm{C}$.

Evaluación de los extractos acuosos de los suplementos vitamínicos sobre la movilidad espermática.

Se realizó la incubación de los extractos acuosos con los espermatozoides en proporción 1 a 1 durante tres horas evaluando la movilidad espermática por microscopía de luz cada 60 minutos. Los resultados son expresados como porcentaje de la movilidad espermática. Como control negativo se usó la muestra total de semen incubada en PBS mientras que como control positivo se utilizó la muestra total de semen incubada con cafeína (Sigma-Aldrich, St. Louis, MO, EEUU) a $4 \mathrm{Mm}$. 


\section{Análisis estadístico}

Se realizó un análisis descriptivo determinando los valores de la media y el rango de la movilidad espermática antes y después de la incubación con cada uno de los extractos. Se realizó un ANOVA de dos vías. Todas las determinaciones se realizaron mediante el programa estadístico GraphPadPrism 7.0 (GraphPad Software, San Diego, CA, EEUU) y se consideró un nivel mínimo de significancia del 95\%, valor $p$ de 0.05 .

\section{Resultados}

El análisis del efecto de los tratamientos con los extractos acuosos de los suplementos vitamínicos sobre los espermatozoides, fueron divididos según la presencia de L-arginina en su composición. No se observaron cambios significativos en la movilidad en función del tiempo a excepción del suplemento Vigorday, en el grupo que tiene
L-arginina, el cual presenta una tendencia en la disminución de la movilidad espermática desde los 60 minutos y el tratamiento con el suplemento Sex Bull en el grupo de vitamínicos que carecen de L-arginina (-Figs. 1 y 2).

\section{Discusión}

Existen estudios en los cuales se utiliza la suplementación oral con la finalidad de mejorar los parámetros seminales convencionales mediante la utilización de combinaciones como L-arginina con Pycnogenol, L-citrulina o roburinas ${ }^{17,18}$ al igual que el uso de plantas medicinales para aumentar la libido y mejorar los parámetros seminales con resultados positivos en humanos, sin embargo, los resultados de la aplicación in vitro de esos suplementos sobre los espermatozoides es discordante, previas investigaciones han utilizado medicamentos de uso oral como el citrato de sildenafil ${ }^{19-22}$ o el tadalafil ${ }^{23}$ con el fin de

\section{Composición: L- Arginina}
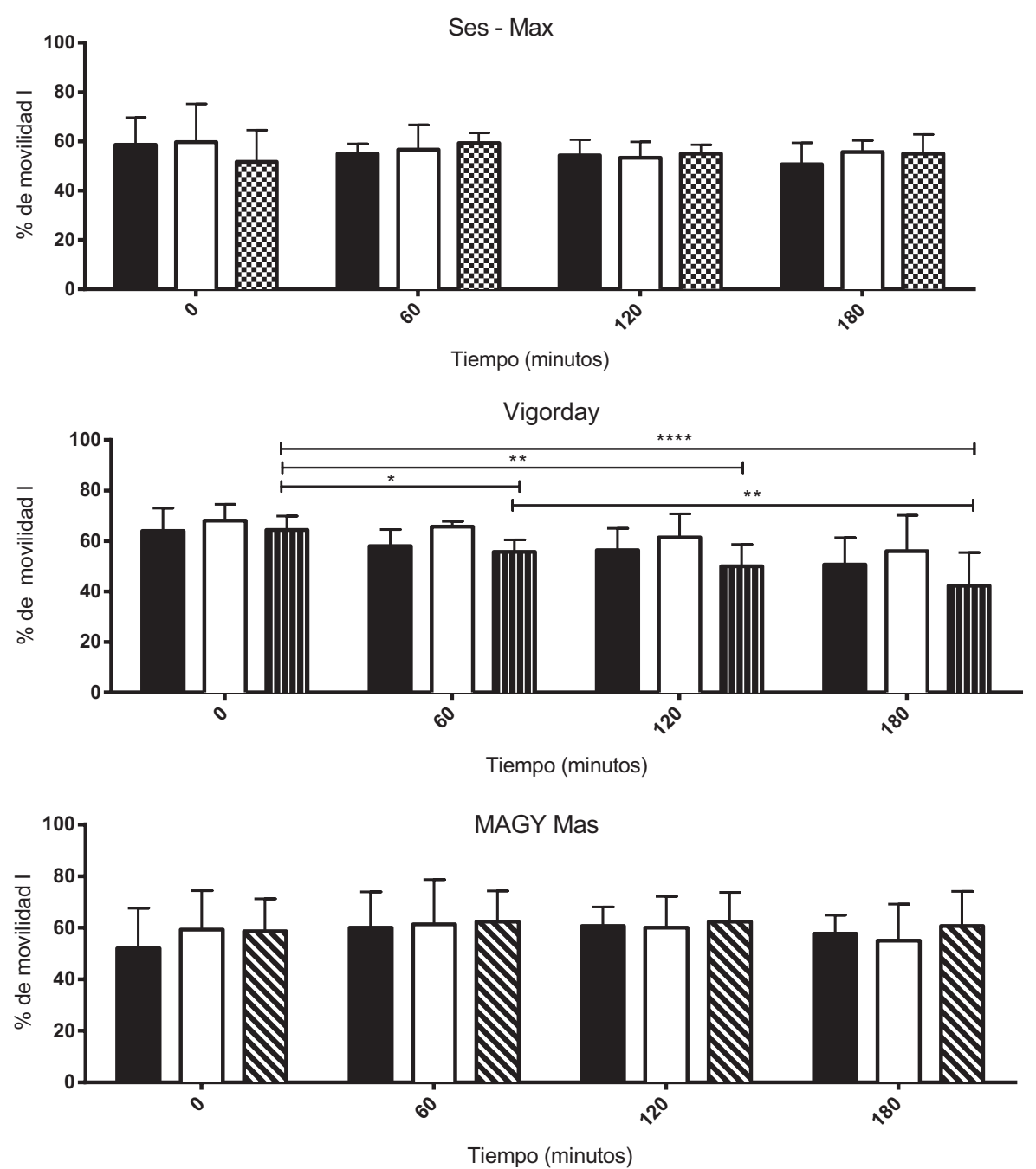

Control negativo

Fig. 1 Evaluación del efecto acuoso de suplementos vitamínicos con L- arginina ( $2 \mathrm{mg} / \mathrm{mL}$ ) en proporción 1:1 sobre la movilidad espermática en función del tiempo (0,60, 120 y 180 minutos). ${ }^{*}$, p $<0.01$; ${ }^{* *}, \mathrm{p}<0.001 ;{ }^{* * * *}, \mathrm{p}<0.0001$. 


\section{Composición: Sin L - Arginina}
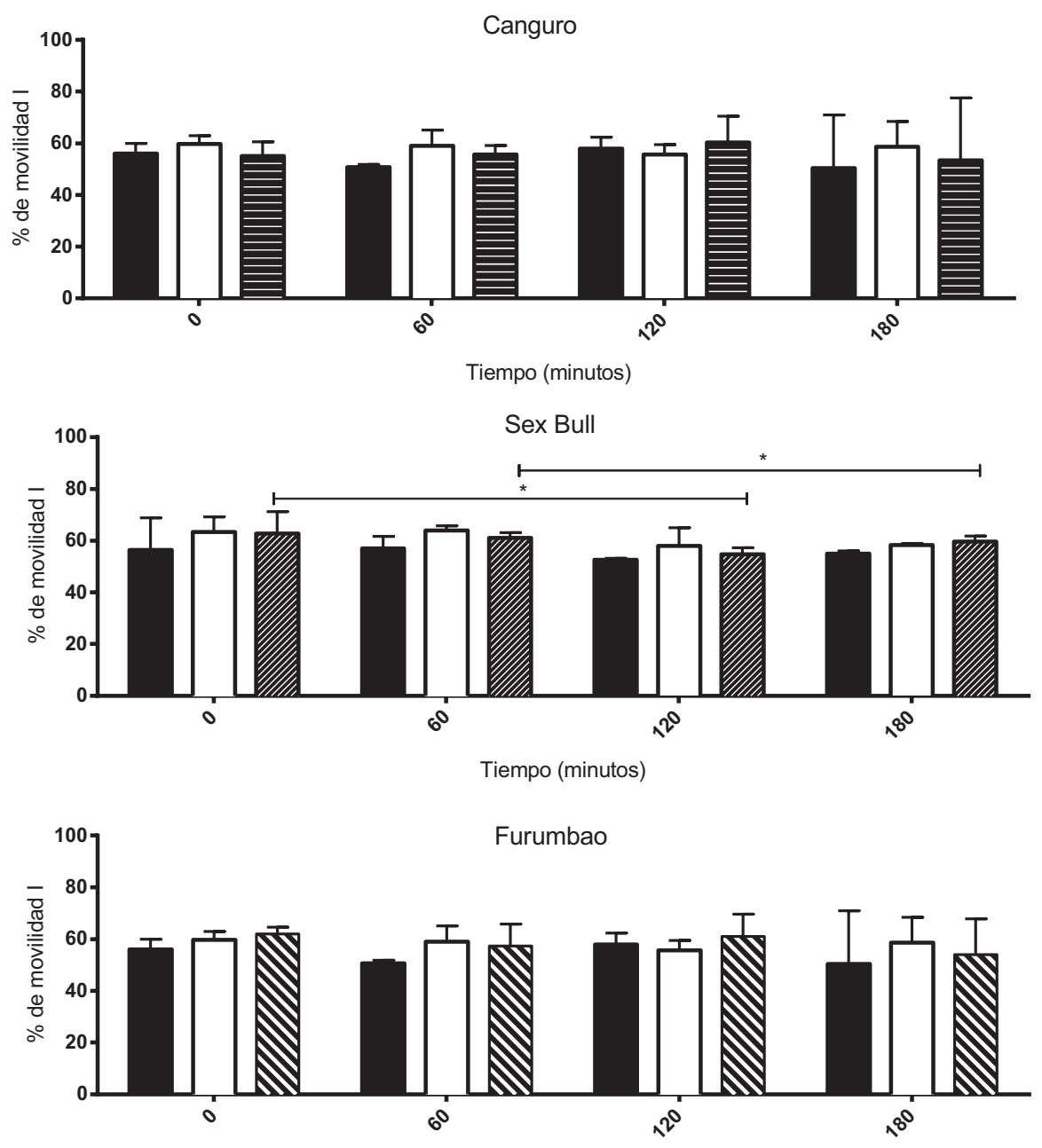

Tiempo (minutos)

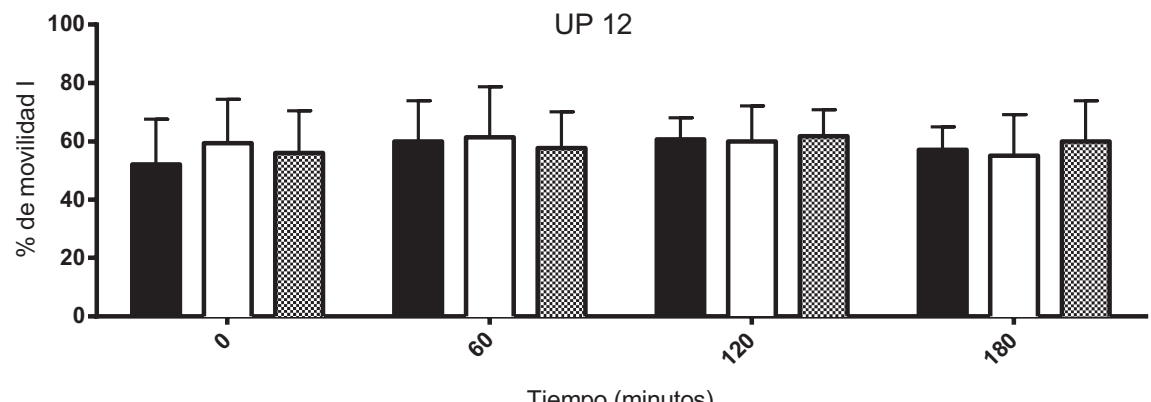

Control negativo

Cafeína

\# Canguro Sex Bull $\quad$ N Furumbao $\approx$ UP 12

Fig. 2 Evaluación del efecto acuoso de suplementos vitamínicos sin L- arginina $(2 \mathrm{mg} / \mathrm{mL})$ en proporción 1:1 sobre la movilidad espermática en función del tiempo $(0,60,120$ y 180 minutos $) .{ }^{*}, \mathrm{p}<0.05$. 
incrementar la movilidad espermática in vitro. Burger y col., incubaron espermatozoides con diferentes concentraciones de Sildenafil $(125,250$ y $750 \mathrm{ng} / \mathrm{mL})$ sin encontrar ningún efecto sobre la movilidad y viabilidad de los espermatozoides respecto al control. ${ }^{19}$ Andrade y col., encontraron que $200 \mu \mathrm{g} / \mathrm{mL}$ de citrato sildenafil afectaba la movilidad, sin embargo, una dosis de $2,000 \mu \mathrm{g} / \mathrm{mL}$ disminuía la movilidad espermática en un $50 \%{ }^{20}$ En el año 2007, Mostafa y col., observó que los espermatozoides tratados con 0,5 y $2 \mathrm{mg} / \mathrm{mL}$ de citrato de sildenafil, aumentaban la movilidad espermática aunque la concentración de $2 \mathrm{mg} / \mathrm{mL}$ no fue estadísticamente significativa. $^{21}$

En el presente estudio, no se observaron cambios significativos en la mayoría de los tratamientos, e incluso dos de los compuestos evaluados (Vigorday y Sex Bull) disminuyeron progresivamente la movilidad de forma significativa, dichos comprimidos están compuestos por plantas afrodisíacas, cuya utilización in vitro ha reportado cambios negativos en la movilidad espermática, por ejemplo Gallego y col., encontró que tres extractos de plantas colombianas (M. platyclada, Z. lenticulare y P. subpedale) disminuyen la movilidad progresiva ${ }^{24}$ al igual que Ospinaet y col., utilizar extractos de frutas afrodisíacas como borojo $(B$. patinoi) y chontduro (E. Cardamomum). ${ }^{11}$

Esos resultados podrían obedecer a que la cantidad de Larginina suministrada en los suplementos vitamínicos induce un aumento en la producción de óxido nítrico por parte de los espermatozoides, produciendo fragmentación de ADN y disminución de la viabilidad y por ende en la movilidad celular, sumado a que se pueden originar compuestos como el peroxinitrito que disminuye el potencial de membrana mitocondrial e inhibe la mayoría de los componentes de la cadena transportadora de electrones, debido a que causa oxidación de la cisteína, la nitración de tirosina y daño a las proteínas hierro-azufre. ${ }^{25}$

\section{Conclusiones}

En conclusión, los suplementos vitamínicos que se venden en las tiendas naturistas y sexuales de la ciudad, no ejercen ningún efecto sobre la calidad seminal in vitro, seguramente basados en su composición solo sirven como suplementos energéticos.

\section{Conflicto de intereses}

Los autores declaran no tener ningún conflicto de intereses.

\section{Protección de personas y animales}

Los autores declaran que los procedimientos seguidos se conformaron a las normas éticas del comité de experimentación humana responsable y de acuerdo con la Asociación Médica Mundial y la Declaración de Helsinki.

\section{Confidencialidad de los datos}

Los autores declaran que han seguido los protocolos de su centro de trabajo sobre la publicación de datos de pacientes.
Derecho a la privacidad y consentimiento informado Los autores han obtenido el consentimiento informado de los pacientes y/o sujetos referidos en el artículo. Este documento obra en poder del autor de correspondencia.

\section{Bibliografía}

1 Thakur M, Chauhan NS, Bhargava S, Dixit VK. A comparative study on aphrodisiac activity of some ayurvedic herbs in male albino rats. Arch Sex Behav 2009;38(06):1009-1015

2 Kotta S, Ansari SH, Ali J. Exploring scientifically proven herbal aphrodisiacs. Pharmacogn Rev 2013;7(13):1-10[Internet]

3 Subramoniam A, Gangaprasad A, Sureshkumar PK, Radhika J, Arun KB. A novel aphrodisiac compound from an orchid that activates nitric oxide synthases. Int J Impot Res 2013;25(06):212-216

4 Sahoo HB, Nandy S, Senapati AK, Sarangi SP, Sahoo SK. Aphrodisiac activity of polyherbal formulation in experimental models on male rats. Pharmacognosy Res 2014;6(02):120-126

5 Medina LO, Forero JE, Maya WC. Plantas afrodisíacas como potenciales capacitantes de espermatozoides humanos. Revista de fitoterapia 2015;15(01):129-136

6 Gonzales GF, Cordova A, Gonzales C, Chung A, Vega K, Villena A. Lepidium meyenii (Maca) improved semen parameters in adult men. Asian J Androl 2001;3(04):301-303

7 Singh AP, Sarkar S, Tripathi M, Rajender S. Mucuna pruriens and its major constituent L-DOPA recover spermatogenic loss by combating ROS, loss of mitochondrial membrane potential and apoptosis. PLoS One 2013;8(01):e54655

8 Melnikovova I, Fait T, Kolarova M, Fernandez EC, Milella L. Effect of Lepidium meyenii Walp. on Semen Parameters and Serum Hormone Levels in Healthy Adult Men: A Double-Blind, Randomized, PlaceboControlled Pilot Study. Evid Based Complement Alternat Med 2015; 2015:324369

9 Lampiao F, Krom D, du Plessis SS. The in vitro effects of Mondia whitei on human sperm motility parameters. Phytother Res 2008; 22(09):1272-1273

10 Tambi MI, Imran MK. Eurycoma longifolia Jack in managing idiopathic male infertility. Asian J Androl 2010;12(03):376-380

11 Ospina Medina L, Pastrana M, Cardona Maya WD. Extractos de frutas afrodisíacas inhiben la movilidad espermática humana in vitro. Rev Plant Med 2018

12 Bhilawadikar R, Zaveri K, Mukadam L, et al. Levels of Tektin 2 and CatSper 2 in normozoospermic and oligoasthenozoospermic men and its association with motility, fertilization rate, embryo quality and pregnancy rate. J Assist Reprod Genet 2013;30(04):513-523

13 WHO. Laboratory manual for examination and processing of human semen. 2010

14 Montanino Oliva M, Poverini R, Lisi R, Carra MC, Lisi F. Treating Woman with Myo-Inositol Vaginal Suppositories Improves Partner's Sperm Motility and Fertility. Int J Endocrinol 2016; 2016:7621942. Doi: 10.1155/2016/7621942

15 Hernández-matos Y, Delgado-roche L, López-pérez R, Martínezsánchez G, Mallok A sobre la fertilidad masculina. 2010;18(3):153-8

16 Saxena A, Prakash P, Porwal M, Sissodia N, Sharma P. Erectile dysfunction: A review and herbs used for its treatment. International Journal of Green Pharmacy 2012;6(02):109

17 Kobori Y, Suzuki K, Iwahata T, et al. Improvement of seminal quality and sexual function of men with oligoasthenoteratozoospermia syndrome following supplementation with L-arginine and Pycnogenol®. Arch Ital Urol Androl 2015;87(03):190-193

18 Stanislavov R, Rohdewald P. Sperm quality in men is improved by supplementation with a combination of L-arginine, L-citrullin, roburins and Pycnogenol ${ }^{\circledR}$. Minerva Urol Nefrol 2014;66(04):217-223

19 Burger M, Sikka SC, Bivalacqua TJ, Lamb DJ, Hellstrom WJ. The effect of sildenafil on human sperm motion and function from normal and infertile men. Int J Impot Res 2000;12(04):229-234 
20 Andrade JR, Traboulsi A, Hussain A, Dubin NH. In vitro effects of sildenafil and phentolamine, drugs used for erectile dysfunction, on human sperm motility. Am J Obstet Gynecol 2000;182(05): 1093-1095

21 Mostafa $T$. In vitro sildenafil citrate use as a sperm motility stimulant. Fertil Steril 2007;88(04):994-996

22 Glenn DR, McVicar CM, McClure N, Lewis SE. Sildenafil citrate improves sperm motility but causes a premature acrosome reaction in vitro. Fertil Steril 2007;87(05):1064-1070
23 Yang Y, Ma Y, Yang $\mathrm{H}$, et al. Effect of acute tadalafil on sperm motility and acrosome reaction: in vitro and in vivo studies. Andrologia 2014;46(04):417-422

24 Gallego G, Henao D, Ospina L, et al. Efecto de cinco extractos de plantas colombianas sobre espermatozoides humanos. Rev Cuba Plantas Med 2012;17(01):84-92

25 Quintero Quinchia YC, Cardona Maya WD. Óxido nítrico y fertilidad masculina: relación directa con los parámetros seminales. Urología Colombiana 2017;26(01):53-60 